\title{
Charge instabilities and topological phases in the extended Hubbard model on the honeycomb lattice with enlarged unit cell
}

\author{
Adolfo G. Grushin, ${ }^{1}$ Eduardo V. Castro, ${ }^{2}$ Alberto Cortijo, ${ }^{1}$ Fernando de Juan, ${ }^{3,4}$ \\ María A. H. Vozmediano, ${ }^{1}$ and Belén Valenzuela ${ }^{1}$ \\ ${ }^{1}$ Instituto de Ciencia de Materiales de Madrid, CSIC, Cantoblanco, E-28049 Madrid, Spain \\ ${ }^{2}$ CFIF, Instituto Superior Técnico, TU Lisbon, Av. Rovisco Pais, 1049-001 Lisboa, Portugal \\ ${ }^{3}$ Lawrence Berkeley National Laboratory, 1 Cyclotron Rd, Berkeley, California 94720, USA \\ ${ }^{4}$ Department of Physics, University of California, Berkeley, California 94720, USA
}

(Received 4 January 2013; published 28 February 2013)

\begin{abstract}
We study spontaneous symmetry breaking in a system of spinless fermions in the honeycomb lattice paying special emphasis to the role of an enlarged unit cell on time reversal symmetry broken phases. We use a tight-binding model with nearest-neighbor hopping $t$ and Hubbard interaction $V_{1}$ and $V_{2}$ and extract the phase diagram as a function of electron density and interaction within a mean-field variational approach. The analysis completes the previous work done in Phys. Rev. Lett. 107, 106402 (2011) where phases with nontrivial topological properties were found with only a nearest-neighbor interaction $V_{1}$ in the absence of charge decouplings. We see that the topological phases are suppressed by the presence of metallic charge density fluctuations. The addition of next to nearest-neighbor interaction $V_{2}$ restores the topological nontrivial phases.
\end{abstract}

DOI: 10.1103/PhysRevB.87.085136

PACS number(s): 71.10.Hf, 11.30.Er, 71.10.Ay

\section{INTRODUCTION}

Topological phases of matter are a new paradigm in condensed matter physics. ${ }^{1-4}$ These phases evade a standard classification in terms of local order parameters and broken symmetries, being described by topological invariants. In addition to the obvious interest from a fundamental viewpoint, the robustness of topological properties against certain local perturbations makes these phases appealing also in applied physics. The recently discovered time reversal invariant topological insulators are a rising star in the family, with topological invariants protected against nonmagnetic disorder by time reversal symmetry $(\mathcal{T}){ }^{5-7}$ Together with time reversal invariant topological insulators, the quantum Hall insulating state is the paradigmatic example of a topological phase. ${ }^{8}$ The nontrivial topology is in this case driven by an external magnetic field that breaks $\mathcal{T}$. In quantum anomalous Hall (QAH) insulators, another example of a topological phase, $\mathcal{T}$ is broken spontaneously, and a quantized anomalous Hall (AH) conductivity arises in the absence of any external magnetic field. ${ }^{9}$ When the Fermi level does not fall into the bulk band gap there is a nonquantized contribution to the AH conductivity characterized as a property of the Fermi surface through its Berry phase and the systems are termed topological Fermi liquids. 1,10

The honeycomb lattice is perhaps the best studied case for its special properties. ${ }^{11,12}$ It is bipartite and yet due to its topology, it is easily amenable to frustration. It is fair to say that the pioneer works proposing this lattice as a "condensed matter simulation of a three-dimensional anomaly," 2,13 together with the analysis of the spin orbit in graphene done in Refs. 14,15 opened the modern field of topological insulators. In these pioneering works, the breaking of time reversal symmetry that allowed the topological nontrivial phases was explicitly put in the Hamiltonian in the form of complex hopping parameters. The noninteracting behavior of the topological phases in the insulating family is at present fairly well understood, and the attention is now shifting to the effect of electron-electron interactions on these phases ${ }^{16-24}$ and to the nature of the phase transitions between topological and ordinary phases (see Ref. 17 and references therein). In most cases, following the original work by Haldane, ${ }^{2}$ breaking $\mathcal{T}$ is associated with bond order (complex hoppings) and originating finite flux states. ${ }^{25-29}$ In this later context, the electron spin is not meant to be a key ingredient, contrary to the topological insulators known to date where the strong spin-orbit coupling is responsible for the nontrivial topology. Focusing in the two-dimensional (2D) case and using spinless fermionic models, the strategy then is to search for spontaneously broken $\mathcal{T}$ phases showing an $\mathrm{AH}$ or $\mathrm{QAH}$ effect driven by Coulomb interactions.

A realization of the Haldane model through electronelectron interactions was obtained at mean-field level in the honeycomb lattice in Refs. 25,29 by adding second-neighbor Coulomb interactions. Other proposals involve more complex lattices that allow for intracell fluxes, as the checkerboard, the kagome, or the decorated honeycomb. ${ }^{26-28} \mathcal{T}$-broken superconducting states have also been proposed recently on the honeycomb lattice. ${ }^{30,31}$

In a recent publication, ${ }^{32}$ we proposed enlarging the unit cell of simple lattice models as an alternative way to drive the spontaneous appearance of phases with broken $\mathcal{T}$. We explored as an example the nearest-neighbor (NN) tightbinding model for spinless fermions interacting through an NN Coulomb interaction in the honeycomb lattice. Our main motivation was the idea that enlarging the unit cell - to enable, for instance, Kekulé type of distortions-would allow for nontrivial topological phases without the necessity to go to longer-range hopping or interactions. We also focused on a region of high doping near the Van Hove singularity where short-range electronic interactions are enhanced and give rise to interesting phases. For this purpose, we considered a minimal model and restricted the mean-field decoupling to order parameters of the type $\left\langle a_{i}^{+} b_{j}\right\rangle$ ignoring possible 
charge-ordered phases with order parameters of the type $\left\langle c_{i}^{+} c_{i}\right\rangle$. The result was that $\mathcal{T}$-broken phases with interesting topological features appeared at large fillings above the van Hove filling for reasonable values of the interaction. The most interesting region of the phase diagram occurred around the commensurate value $n=1$ corresponding to having one electron per enlarged ( $\mathrm{six}$ atoms) unit cell. There, the system was insulating above a critical value of the NN Hubbard interaction $V \equiv V_{1}$. In this work, we complete the former analysis by allowing charge decouplings in the mean-field equations. We find that charge inhomogeneous phases dominate the phase diagram in the region where $\mathcal{T}$ broken phases occurred. $\mathcal{T}$ broken phases reemerge and are stabilized by the addition of a next nearest-neighbor (NNN) interaction $V_{2}$. The results then are similar to these obtained at half-filling in the pioneer work of Ref. 25 in the sense that $V_{2} \neq 0$ is needed to stabilize nontrivial phases. In the present work, however, we have found that the high-doping $\mathcal{T}$-broken phases are stabilized for $V_{2} \ll V_{1}$, while at half-filling $V_{2}>V_{1}$ is required.

The paper is organized as follows. In Sec. II, we describe the model and the method of calculation. In Sec. III, we explain the phase diagram where for completeness, and to compare with previous results, we present also the situation at half-filling (see Sec. III A). In Sec. III B, we analyze the modification introduced in the $V_{2}=0$ case by the charge decoupling at higher fillings. We will see that the charge modulated phases wash out the topologically nontrivial phases. Finally, we see how these are restored by the inclusion of the second-neighbor interaction and describe the $\mathcal{T}$ broken phases and their band structure. In Sec. IV, we summarize the situation and discuss some open problems. Appendix contains the technical details of the calculation.

\section{MODEL}

We consider the model describing spinless electrons on the honeycomb lattice,

$$
H=-t \sum_{\langle i, j\rangle} c_{i}^{\dagger} c_{j}+V_{1} \sum_{\langle i, j\rangle} n_{i} n_{j}+V_{2} \sum_{\langle\langle i, j\rangle\rangle} n_{i} n_{j},
$$

where $t$ is the $\mathrm{NN}$ hopping, and $V_{1}$ and $V_{2}$ the $\mathrm{NN}$ and NNN repulsions, all of them real. The operator $c_{i}^{\dagger}\left(c_{i}\right)$ creates (annihilates) a fermion at site $i$, the number operator is $n_{i}=c_{i}^{\dagger} c_{i}$, and the sums run over either NN sites $\langle i, j\rangle$ or NNN sites $\langle\langle i, j\rangle\rangle$.

We use a six-atom unit cell to allow for finite flux also in NN loops and not only in NNN loops. The basis vectors can be chosen as $\mathbf{a}_{1}=\frac{3 a}{2}(-\sqrt{3}, 1)$ and $\mathbf{a}_{2}=\frac{3 a}{2}(\sqrt{3}, 1)$, and their counterparts in reciprocal space are $\mathbf{b}_{1}=\frac{2 \pi}{3 \sqrt{3} a}(-1, \sqrt{3})$ and $\mathbf{b}_{2}=\frac{2 \pi}{3 \sqrt{3} a}(1, \sqrt{3})$. The number of Fourier components of local operators is six, $a_{\iota, \mathbf{k}}^{\dagger}=\frac{1}{\sqrt{N}} \sum_{i \in A \iota} c_{i}^{\dagger} e^{i \mathbf{k} \cdot \mathbf{r}_{i}}$ and $b_{\iota, \mathbf{k}}^{\dagger}=$ $\frac{1}{\sqrt{N}} \sum_{i \in B \iota} c_{i}^{\dagger} e^{i \mathbf{k} \cdot \mathbf{r}_{i}}$, with $\iota=1,2,3$. In terms of the new operators, the Hamiltonian in Eq. (1) reads as

$$
\begin{aligned}
H= & -t \sum_{\mathbf{k}} a_{i, \mathbf{k}}^{\dagger}\left(\gamma_{\mathbf{k}}^{i j}\right)^{*} b_{j, \mathbf{k}}+\text { H.c. } \\
& +\frac{V_{1}}{N} \sum_{\mathbf{k}, \mathbf{k}^{\prime}, \mathbf{q}} a_{i, \mathbf{k}}^{\dagger} a_{i, \mathbf{k}-\mathbf{q}} \gamma_{\mathbf{q}}^{i j} b_{j, \mathbf{k}^{\prime}}^{\dagger} b_{j, \mathbf{k}^{\prime}+\mathbf{q}} \\
& +\frac{V_{2}}{2 N} \sum_{\mathbf{k}, \mathbf{k}^{\prime}, \mathbf{q}} a_{i, \mathbf{k}}^{\dagger} a_{i, \mathbf{k}-\mathbf{q}} \alpha_{\mathbf{q}}^{i j} a_{j, \mathbf{k}^{\prime}}^{\dagger} a_{j, \mathbf{k}^{\prime}+\mathbf{q}} \\
& +\frac{V_{2}}{2 N} \sum_{\mathbf{k}, \mathbf{k}^{\prime}, \mathbf{q}} b_{i, \mathbf{k}}^{\dagger} b_{i, \mathbf{k}-\mathbf{q}} \beta_{\mathbf{q}}^{i j} b_{j, \mathbf{k}^{\prime}}^{\dagger} b_{j, \mathbf{k}^{\prime}+\mathbf{q}},
\end{aligned}
$$

where summation over repeated indices is assumed (thus the factor $1 / 2$ in the last two terms), with $i, j=1,2,3$, and where we have defined the $3 \times 3$ matrices

$$
\begin{aligned}
& \boldsymbol{\gamma}_{\mathbf{q}}=\left[\begin{array}{ccc}
1 & e^{-i \mathbf{a}_{2} \cdot \mathbf{q}} & 1 \\
1 & 1 & e^{i\left(\mathbf{a}_{1}+\mathbf{a}_{2}\right) \cdot \mathbf{q}} \\
e^{-i \mathbf{a}_{1} \cdot \mathbf{q}} & 1 & 1
\end{array}\right] \\
& \boldsymbol{\alpha}_{\mathbf{q}}=\left[\begin{array}{ccc}
0 & 1+e^{i \mathbf{q} \cdot\left(\mathbf{a}_{1}+\mathbf{a}_{2}\right)}+e^{i \mathbf{q} \cdot \mathbf{a}_{2}} & 1+e^{-i \mathbf{q} \cdot \mathbf{a}_{1}}+e^{i \mathbf{q} \cdot \mathbf{a}_{2}} \\
1+e^{-i \mathbf{q} \cdot\left(\mathbf{a}_{1}+\mathbf{a}_{2}\right)}+e^{-i \mathbf{q} \cdot \mathbf{a}_{2}} & 0 & 1+e^{-i \mathbf{q} \cdot\left(\mathbf{a}_{1}+\mathbf{a}_{2}\right)}+e^{-i \mathbf{q} \cdot \mathbf{a}_{1}} \\
1+e^{i \mathbf{q} \cdot \mathbf{a}_{1}}+e^{-i \mathbf{q} \cdot \mathbf{a}_{2}} & 1+e^{i \mathbf{q} \cdot\left(\mathbf{a}_{1}+\mathbf{a}_{2}\right)}+e^{i \mathbf{q} \cdot \mathbf{a}_{1}} & 0
\end{array}\right], \\
& \boldsymbol{\beta}_{\mathbf{q}}=\left[\begin{array}{ccc}
0 & 1+e^{-i \mathbf{q} \cdot \mathbf{a}_{1}}+e^{i \mathbf{q} \cdot \mathbf{a}_{2}} & 1+e^{-i \mathbf{q} \cdot\left(\mathbf{a}_{1}+\mathbf{a}_{2}\right)}+e^{-i \mathbf{q} \cdot \mathbf{a}_{1}} \\
1+e^{i \mathbf{q} \cdot \mathbf{a}_{1}}+e^{-i \mathbf{q} \cdot \mathbf{a}_{2}} & 0 & 1+e^{-i \mathbf{q} \cdot\left(\mathbf{a}_{1}+\mathbf{a}_{2}\right)}+e^{-i \mathbf{q} \cdot \mathbf{a}_{2}} \\
1+e^{i \mathbf{q} \cdot\left(\mathbf{a}_{1}+\mathbf{a}_{2}\right)}+e^{i \mathbf{q} \cdot \mathbf{a}_{1}} & 1+e^{i \mathbf{q} \cdot\left(\mathbf{a}_{1}+\mathbf{a}_{2}\right)}+e^{i \mathbf{q} \cdot \mathbf{a}_{2}} & 0
\end{array}\right]
\end{aligned}
$$

Note that while $\boldsymbol{\alpha}_{\mathbf{q}}$ and $\boldsymbol{\beta}_{\mathbf{q}}$ are Hermitian, the matrix $\boldsymbol{\gamma}_{\mathbf{q}}$ is not.

\section{PHASE DIAGRAM}

The phase diagram of the model is obtained within the variational mean-field approach. The details of the mean-field decoupling and related equations are extensively explained in
Appendix. In brief, we replace the four fermion interaction terms in Eq. (2) with bilinears, which, when written in real space, can be interpreted as the most general hopping and potential energy terms compatible with the reduced translational symmetry of the lattice (6 atom unit cell). In total, there are nine NN $(\xi)$ and $18 \mathrm{NNN}$ (nine per sublattice, $\chi^{A}$ and $\chi^{B}$ ) complex hopping parameters, which are depicted 

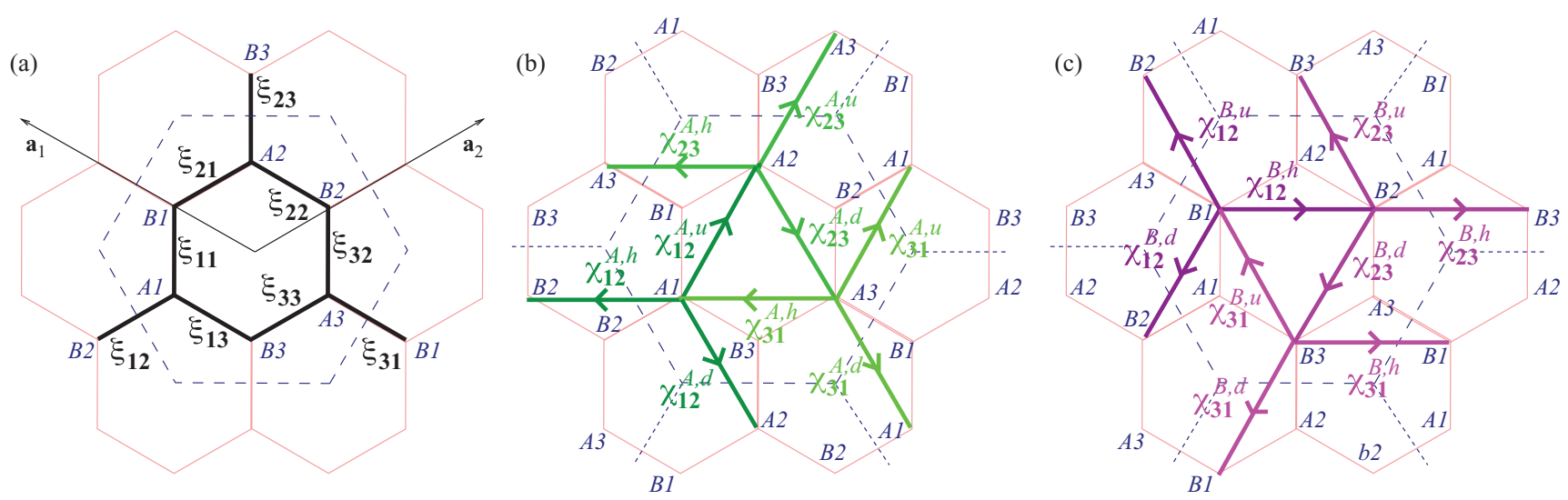

FIG. 1. (Color online) Unit cell and mean-field parameters of our model. In each panel we show 9 distinct effective hoppings, making up a total of 27 (see Appendix A 1).

in Fig. 1. In addition, there are six local energy terms (three per sublattice, $\rho^{A}$ and $\rho^{B}$ ), of which only five are independent due to charge conservation. See Appendix A 1 for details.

Using the variational mean-field approach, one finds the set of $33=3 \times 9+6$ mean-field equations that, complemented by charge conservation, determine the mean-field parameters and the chemical potential $\mu$ (see Appendixes A 2 and A 3). The mean-field equations read as

$$
\begin{aligned}
\xi_{i j} & =-\frac{V_{1}}{N} \sum_{\mathbf{k}} \gamma_{\mathbf{k}}^{i j}\left\langle b_{j}^{\dagger}(\mathbf{k}) a_{i}(\mathbf{k})\right\rangle_{\mathrm{MF}}, \\
\chi_{i j}^{A, \delta} & =-\frac{V_{2}}{N} \sum_{\mathbf{k}} \lambda_{\mathbf{k}, i j}^{A, \delta}\left\langle a_{j}^{\dagger}(\mathbf{k}) a_{i}(\mathbf{k})\right\rangle_{\mathrm{MF}}, \\
\chi_{i j}^{B, \delta} & =-\frac{V_{2}}{N} \sum_{\mathbf{k}} \lambda_{\mathbf{k}, i j}^{B, \delta}\left\langle b_{j}^{\dagger}(\mathbf{k}) b_{i}(\mathbf{k})\right\rangle_{\mathrm{MF}}, \\
\rho_{i}^{A} & =V_{1} n_{B}+3 V_{2} n_{A}-3 V: 2 n_{i}^{A}, \\
\rho_{i}^{B} & =V_{1} n_{A}+3 V_{2} n_{B}-3 V_{2} n_{i}^{B},
\end{aligned}
$$

where $\lambda_{\mathbf{k}, i j}^{A, \delta}, \lambda_{\mathbf{k}, i j}^{B, \delta}$ are phase factors analogous to $\gamma_{k}^{i j}$ defined in Eq (3), $n_{i}^{c}=\frac{1}{N} \sum_{k}\left\langle c_{i}^{\dagger}(\mathbf{k}) c_{i}(\mathbf{k})\right\rangle_{\mathrm{MF}}$ and $n_{c}=\sum_{i=1}^{3^{k}} n_{i}^{c}$ with $c=A, B$. Detailed expressions for these matrices can be found in Appendix A2. The notation $\langle\ldots\rangle_{\mathrm{MF}}$ means average in the macrocanonical ensemble taking the mean-field Hamiltonian in the Boltzmann factor.

In order to obtain the mean-field phase diagram, we solve the mean-field equations self-consistently (see Appendix A 3) and take the solution (if more than one is obtained) that minimizes the free energy in Eq. (A10) (see Appendix A 4). Care must be taken with charge-like order parameters, Eqs. (9) and (10). Due to the frustration introduced by NNN interaction, these order parameters may flow to a non-self-consistent solution where the charge-like order parameters in different sublattices interchange at each step. Apart from this subtlety, getting a solution is straightforward.

We will analyze first the phase diagram obtained at halffilling, which is interesting on its own and later discuss the modification introduced in the $V_{2}=0$ case by the charge decoupling. We will see that the charge modulated phases wash out the topologically nontrivial phases. Finally, we see how these are restored by the inclusion of the second-neighbor interaction.

\section{A. Half-filling}

Let us first analyze the half-filled case, where $n \equiv n_{A}+$ $n_{B}-3=0$. This case provides a test to the present mean-field analysis, since a similar approach, also using a 6-atom unit cell, has been taken in Ref. 29. For comparison, we show the phase diagram obtained in Ref. 29 in the left panel of Fig. 2. In the right panel of Fig. 2, we can see the phase diagram of the present work (we use the same color code). We plot the different phases (that will be described in what follows) as a function of the interaction strength $V_{1}$ and $V_{2}$ in units of the hopping parameter $t$. The half-filled case was first explored in the original lattice in Ref. 25 and nontrivial topological phases were already encountered for values of the interaction $V_{2}>V_{1}$.

For $V_{1} \lesssim 1.5 t$ and $V_{2} \lesssim 2 t$, the two phase diagrams coincide. For $V_{1} \gtrsim 1.5 t$, however, we find that the semimetallic (SM) and the charge density wave (CDW) phases are robust against the Kekule phase. The Kekulé phase is characterized by an alternating bond strength as shown schematically in the inset of the left-hand side of Fig. 3. This distortion is important in the physics of graphene because it opens a gap in the spectrum breaking the translational symmetry of the original honeycomb lattice while preserving time reversal $(\mathcal{T})$
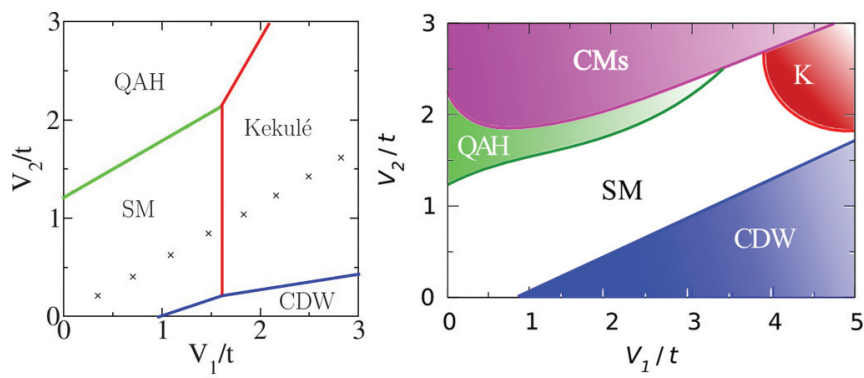

FIG. 2. (Color online) (Left) Mean-field phase diagram for the half-filling case reproduced from Ref. 29. The various phases are described in the text. SM means semimetal. (Right) Mean-field phase diagram obtained in present work. Lines are guides to the eyes. CMs stands for the charge modulated phase discussed in the text. 

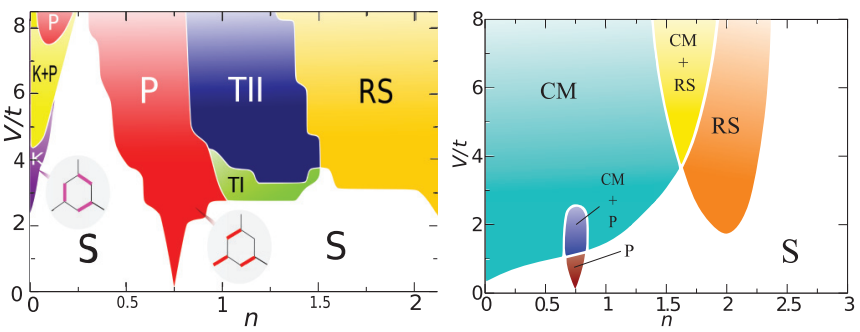

FIG. 3. (Color online) (Left) Phase diagram with $V_{2}=0$ when charge inhomogeneous phases are not allowed. Legend: (S) symmetric phase, i.e., bare graphene with a uniform renormalization of the hopping; (K) Kekule distortion with hopping renormalization as shown in the inset; (P) Pomeranchuk distortion of the Fermi surface and hopping renormalization as shown in the inset; $(\mathrm{K}+\mathrm{P})$ coexistence of Kekule and Pomeranchuk distortions; (TI) and (TII) $\mathcal{T}$ broken phases discussed at length in the text; (RS) broken symmetry state with real hopping parameters, the distortion is neither Kekule type nor Pomeranchuk (reduced symmetry). (Right) Phase diagram with $V_{2}=0$ and charge inhomogeneous phases allowed. CM stands for the new charge modulated phase discussed in the text. In the $\mathrm{CM}+\mathrm{RS}$ phase there is also a real, asymmetric renormalization of the hoppings.

and inversion $(\mathcal{I})$ symmetries. ${ }^{33,34}$ It also plays a key role in the models of charge fractionalization in the honeycomb lattice. ${ }^{35}$ In our approach, the Kekulé phase only appears at much higher $V_{1}$ and $V_{2}$. For $V_{2} \gtrsim 2 t$, a new phase sets in, not predicted in Ref. 29. This is a charge density wave with reduced rotational symmetry; to distinguish from CDW, we denote it as charge modulated with modulation also over the sublattice (CMs). In the $\mathrm{CDW}$, there is a charge imbalance between sublattices, but no inhomogeneity over the sublattice: the charge-like order parameters $\left(\rho_{1}^{A}, \rho_{1}^{B}, \rho_{2}^{A}, \rho_{2}^{B}, \rho_{3}^{A}, \rho_{3}^{B}\right)$ take the form $(\rho,-\rho, \rho,-\rho, \rho,-\rho)$. For CMs, however, the charge is modulated also over the sublattice and the charge-like order parameters take the form $(\rho,-\rho, \rho,-\rho,-\rho-\Delta, \rho+\Delta)$. We note that for $V_{2} \gtrsim V_{1}$ such modulation is naturally expected from a classical point of view (remember that the Hartree contribution has a classical interpretation): the staggered charge modulation of CDW minimizes the energy coming from NN repulsion, but it does not affect the NNN contribution; for $V_{2} \gtrsim V_{1}$ it becomes energetically favorable to reverse a $\mathrm{NN}$ dimmer, paying the corresponding $\mathrm{NN}$ energy $\propto V_{1}$, but reducing the NNN energetic contribution $\propto V_{2}$. Such inhomogeneous charge modulation over the sublattice was not allowed in Ref. 29, and that is the reason why the QAH phase dominates over a larger region of the phase diagram.

\section{B. Higher filling}

We will now discuss the results obtained for higher dopings. The doped system has been the subject of attention recently due to the experimental availability ${ }^{36}$ and to the interesting phases that emerge near the van Hove filling. ${ }^{31,37-39}$ In Ref. 32, we have shown that for the Hamiltonian in Eq. (1) with $V_{2}=0$, and ignoring charge inhomogeneous phases, $\mathcal{T}$-broken phases show up in the phase diagram $V$ versus $n$. The phase diagram is shown in Fig. 3 (left), where the meaning of the various phases is described. The dominant phases in the region of interest above the van Hove filling ( $n=0.75$ in our units)

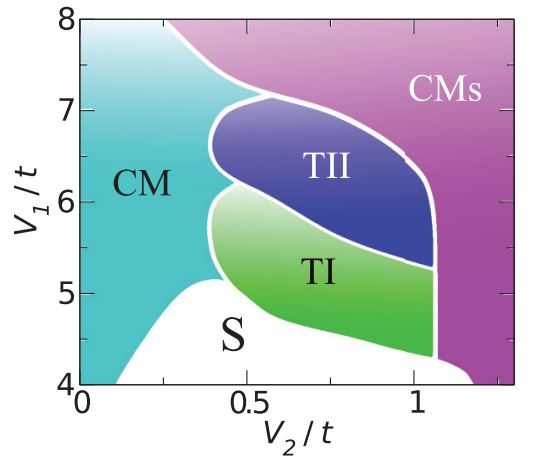

FIG. 4. (Color online) Phase diagram in the plane $V_{1}$ vs $V_{2}$ at $n=1.2$

were a Pomeranchuk instability $(\mathrm{P})$ characterized by rotational symmetry breaking as indicated in the inset, and the $\mathcal{T}$-broken phases denoted by TI and TII obtained at $1 \lesssim n \lesssim 1.5$ and $V_{1} \gtrsim 2 t$. These phases are the same as those obtained in Ref. 32.

When charge inhomogeneous phases are allowed, we obtain the phase diagram shown in Fig. 3 (right). As can be seen, the $\mathcal{T}$-broken phases are washed out by a charge modulated (CM) phase. This charge modulation corresponds to a charge imbalance between the two sublattices, and is homogeneous over the sublattice; at half-filling, this is the CDW discussed in the previous section. This modulation induces a trivial gap between bands 3 and 4 at the $\Gamma$ point. The system is thus a trivial insulator at $n=0$, where the Fermi level falls into the gap, and is metallic for $n>0$. We see that the $\mathrm{CM}$ phase dominates the region of the phase diagram where Pomeranchuk and $\mathcal{T}$-broken phases were stabilized before (left hand side of Fig. 3). The charge decoupling leaves only a small region around the van Hove filling $(n=0.75)$ and for very small values of the interaction where the Pomeranchuk instability is still the most favorable phase. At larger values of the interaction $(\mathrm{CM}+\mathrm{P})$, the charge modulated phase is accompanied of a renormalization of the hopping with the Pomeranchuk symmetry. This is similar to the $(\mathrm{CM}+\mathrm{RS})$ phase that occurs in the phase diagram around $n=0.75$ for larger values of the doping and interaction. A natural way to restore the topologically nontrivial phases is to add an NNN interaction $V_{2}$. Since we are looking for topological phases we have centered our attention in the doping region around the commensurate value $n=1$.

In Fig. 4, we show the phase diagram for $V_{2}>0$ obtained at fixed $n=1.2$. Increasing $V_{2}$, frustrates the CM phase, and $\mathcal{T}$-broken phases are recovered. We note that this happens already for $V_{2} \ll V_{1}$. If $V_{2}$ is further increased, the system falls into the CMs phase (charge modulated with modulation also over the sublattice) already encountered at half-filling, which was discussed in the previous section.

The new $\mathcal{T}$-broken phases TI and TII are similar to the ones described in Ref. 32. Figure 5 shows the real space hopping pattern for TI and TII, respectively. The direction of the arrows represents the sign of the phase of the given complex hopping. As discussed in Ref. 10, the possibility of having nontrivial topological phases characterized by a finite Hall conductivity in $\mathcal{T}$-broken systems is determined by the discrete symmetries $\mathcal{T}$ and space inversion $\mathcal{I}$-preserved in the 

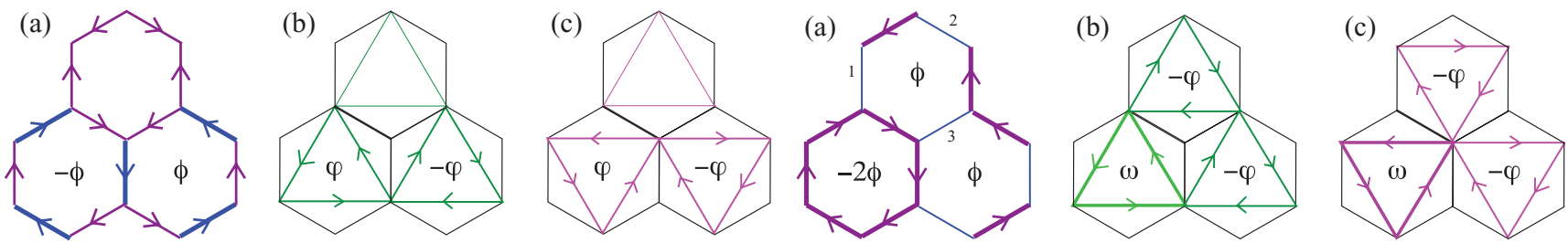

FIG. 5. (Color online) (Left) Flux pattern in the TI phase. (a) The case with $V_{2}=0$ and no charge decoupling in the phase diagram at the left-hand side of Fig. 3. (b) and (c) Flux patterns for the TI phase in Fig. 4. (Right) Same for the TII phase.

system. As happened in the case discussed in Ref. 32, the TII phase is still invariant under inversion hence breaking the product $\mathcal{T}$. $\mathcal{I}$ and is in principle topologically nontrivial with finite Hall conductivity. ${ }^{10}$

Figure 6 shows a typical band structure for the TI and TII phases. The TI case is shown in Fig. 6 (left), where as an example we took the point $V_{1}=5 t$ and $V_{2}=0.75 t$. In the right panel of Fig. 6, we show the TII case, for $V_{1}=6 t$ and $V_{2}=$ $0.75 t$. The two figures are very similar to their counterparts when $V_{2}=0 .{ }^{32}$ The interesting feature is that, unlike what happened there, we do not have any insulating phase away of half-filling, even for the commensurate value $n=1$ whose band structure is very similar to these in Fig. 6 .

\section{CONCLUSIONS AND DISCUSSION}

One of the important points of this and the related work in Ref. 32 is the proposal that nontrivial topological phases can be spontaneously generated from interactions in lattice fermionic systems when translational symmetry is relaxed, i.e., when phases with enlarged unit cells are allowed. The employment of enlarged unit cell allows for novel intracell
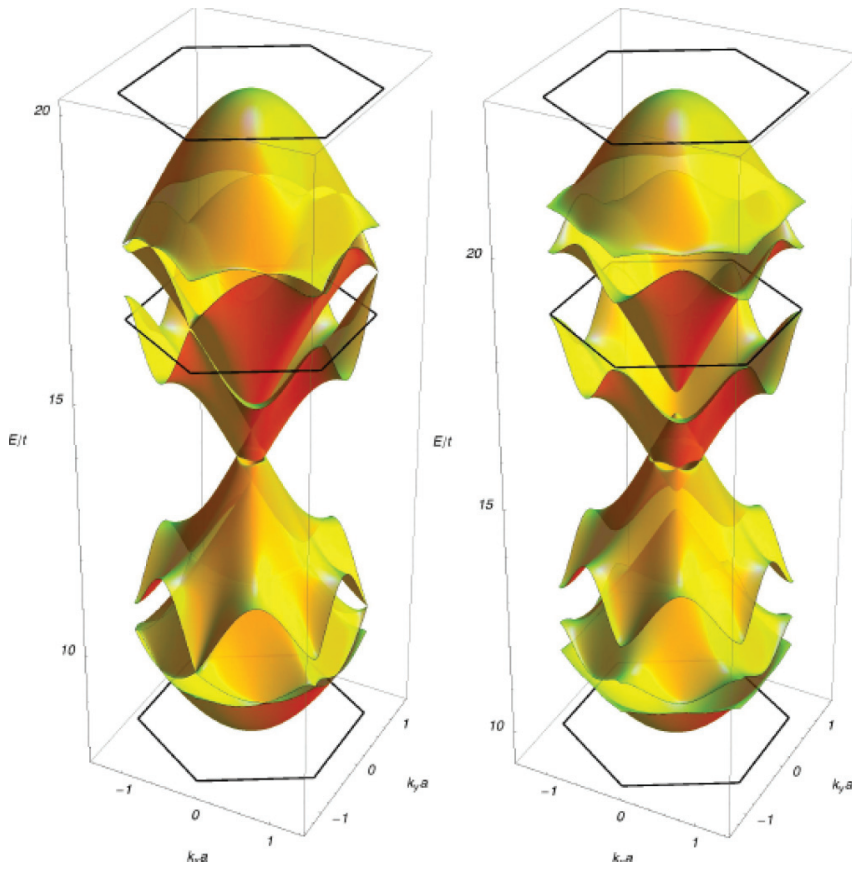

FIG. 6. (Color online) Mean-field band structure in the TI phase for $V_{1}=5 t$ and $V_{2}=0.75 t$ (left) and in the TII phase for $V_{1}=6 t$ and $V_{2}=0.75 t$ (right). The middle black hexagon indicates the position of the Fermi level. current patterns emerging from interactions, and thus novel topological phases. We used the honeycomb lattice with extended Hubbard interaction to exemplify the type of new physics expected. In Ref. 32 , we checked ${ }^{40}$ that no topological phase can arise in the original lattice (two atoms unit cell) without including second nearest-neighbor interactions $V_{2}$ to allow for the formation of the necessary current loops that break time-reversal symmetry.

We have completed the analysis of Ref. 32 including a charge decoupling order parameter. The phase diagram is dominated by charge modulated phases with no particular symmetry and no topological phases were found. The charge modulated phases in the case $V_{2}=0$ are usual CDW phases but we keep the name $\mathrm{CM}$ to emphasize the fact that, away from half-filling, these phases are metallic. When $V_{2} \neq 0$, a different charge modulated phase arises, which we denote CMs in which the charge is modulated also over the sublattice and the charge-like order parameters take the form $(\rho,-\rho, \rho,-\rho,-\rho-\Delta, \rho+\Delta)$. An interesting analysis of charge modulated phases in graphene has been done recently in Ref. 41.

In the previous analysis, ${ }^{32}$ there were two special filling values apart from half-filling: the van Hove filling and the commensurate value $n=1$ corresponding to four electrons per enlarged unit cell. Around the VH density, the system supported a Pomeranchuk deformation and the topological phases were established around the commensurate $n=1$ filling. The system was gapped only at this particular value. In that case, we observed a phase separation in the region in the phase space of fillings in between these two values with the two extremes being the stable phases. ${ }^{40}$ We have been seeing that in the charge modulated phase dominating the phase diagram, the $n=1$ case is not special any more; even though bands 3 and 4 might be nontrivial now (because of $V_{2}$ ), the system at $n=1$ is not an insulator: no "full" gap develops as the Fermi level always crosses bands 4 and 5 (see Fig. 6). This behavior can be understood from the strong coupling point of view due to the frustration induced by the competition between $V_{1}$ and $V_{2}$ for the ground state. The situation is similar to the quarter filling case in one ${ }^{42}$ and two dimensions ${ }^{43}$ where the long-range Coulomb interaction enhances the metallic behavior. Since this is only a mean-field analysis, it will be interesting to check the stability of this phases under quantum fluctuations.

Inclusion of an extra coupling to next to nearest neighbors $V_{2}$, restores the topological phases at fillings around $n=1$. This model was already studied in the literature, in particular in Ref. 25 where topological phases were found at half-filling for values of the interactions $V_{2}>V_{1}$. Allowing charge decoupling that gives rise to the inhomogeneous charge 
modulation over the sublattice described along this work, favors the $\mathcal{T}$-broken phases that now set at lower values of $V_{2}$.

The possibility of superconducting phases have been left out in this analysis. Considering superconducting order parameters embedded in enlarged unit cells might make novel topological superconducting phases emerge, ${ }^{7}$ opening new routes to realize these, sometimes elusive, phases of matter. Very recent works suggest the formation of an eight-atom unit cell spin density wave close to the van Hove filling ( $n \sim 0.75$ in our notation), ${ }^{44}$ or topological superconducting order exactly at $n=0.75 .{ }^{31}$ Whether or not these phases dominate close to $n \sim 1$ or rather a spin Hall effect is energetically more stable is still an open question. The $\mathcal{T}$-broken phases described in this work can be difficult to observe in graphene since they occur at high values of the interaction and filling but they can probably be tested in cold atom experiments with optical lattices. ${ }^{45-49}$

\section{ACKNOWLEDGMENTS}

This research was supported in part by the Spanish MECD grants FIS2011-23713, FIS2011-29689, PIB2010BZ-00512. F. de J. acknowledges support from the "Programa Nacional de Movilidad de Recursos Humanos” (Spanish MECD).

\section{APPENDIX : MEAN-FIELD ANALYSIS}

\section{Mean-field decoupling}

The mean-field Hamiltonian we propose is

$$
H_{\mathrm{MF}}=H_{0}+\sum_{\mathbf{k}} \psi_{\mathbf{k}}^{\dagger}\left(\begin{array}{cccccc}
\rho_{1}^{A} & \xi_{11} & \chi_{12}^{A}(\mathbf{k}) & e^{i \mathbf{k} \cdot \mathbf{a}_{2}} \xi_{12} & \left(\chi_{31}^{A}(\mathbf{k})\right)^{*} & \xi_{13} \\
\xi_{11}^{*} & \rho_{1}^{B} & \xi_{21}^{*} & \chi_{12}^{B}(\mathbf{k}) & e^{-i \mathbf{k} \cdot \mathbf{a}_{1}} \xi_{31}^{*} & \left(\chi_{31}^{B}(\mathbf{k})\right)^{*} \\
\left(\chi_{12}^{A}(\mathbf{k})\right)^{*} & \xi_{21} & \rho_{2}^{A} & \xi_{22} & \chi_{23}^{A}(\mathbf{k}) & e^{\left.-i \mathbf{k} \cdot \mathbf{a}_{1}+\mathbf{a}_{2}\right)} \xi_{23} \\
e^{-i \mathbf{k} \cdot \mathbf{a}_{2}} \xi_{12}^{*} & \left(\chi_{12}^{B}(\mathbf{k})\right)^{*} & \xi_{22}^{*} & \rho_{2}^{B} & \xi_{32}^{*} & \chi_{23}^{B}(\mathbf{k}) \\
\chi_{31}^{A}(\mathbf{k}) & e^{i \mathbf{k} \cdot \mathbf{a}_{1}} \xi_{31} & \left(\chi_{23}^{A}(\mathbf{k})\right)^{*} & \xi_{32} & \rho_{3}^{A} & \xi_{33} \\
\xi_{13}^{*} & \chi_{31}^{B}(\mathbf{k}) & e^{i \mathbf{k} \cdot\left(\mathbf{a}_{1}+\mathbf{a}_{2}\right)} \xi_{23}^{*} & \left(\chi_{23}^{B}(\mathbf{k})\right)^{*} & \xi_{33}^{*} & \rho_{3}^{B}
\end{array}\right) \psi_{\mathbf{k}},
$$

with

$$
\begin{aligned}
& \chi_{12}^{A}(\mathbf{k})=\chi_{12}^{A, u}+e^{i \mathbf{k} \cdot\left(\mathbf{a}_{1}+\mathbf{a}_{2}\right)} \chi_{12}^{A, d}+e^{i \mathbf{k} \cdot \mathbf{a}_{2}} \chi_{12}^{A, h}, \\
& \chi_{23}^{A}(\mathbf{k})=e^{-i \mathbf{k} \cdot\left(\mathbf{a}_{1}+\mathbf{a}_{2}\right)} \chi_{23}^{A, u}+\chi_{23}^{A, d}+e^{-i \mathbf{k} \cdot \mathbf{a}_{1}} \chi_{23}^{A, h}, \\
& \chi_{31}^{A}(\mathbf{k})=e^{-i \mathbf{k} \cdot \mathbf{a}_{2}} \chi_{31}^{A, u}+e^{i \mathbf{k} \cdot \mathbf{a}_{1}} \chi_{31}^{A, d}+\chi_{31}^{A, h},
\end{aligned}
$$

and

$$
\begin{aligned}
& \chi_{12}^{B}(\mathbf{k})=\chi_{12}^{B, h}+e^{i \mathbf{k} \cdot \mathbf{a}_{2}} \chi_{12}^{B, d}+e^{-i \mathbf{k} \cdot \mathbf{a}_{1}} \chi_{12}^{B, u}, \\
& \chi_{23}^{B}(\mathbf{k})=e^{-i \mathbf{k} \cdot \mathbf{a}_{2}} \chi_{23}^{B, h}+\chi_{23}^{B, d}+e^{-i \mathbf{k} \cdot\left(\mathbf{a}_{1}+\mathbf{a}_{2}\right)} \chi_{23}^{B, u}, \\
& \chi_{31}^{B}(\mathbf{k})=e^{i \mathbf{k} \cdot \mathbf{a}_{1}} \chi_{31}^{B, h}+e^{i \mathbf{k} \cdot\left(\mathbf{a}_{1}+\mathbf{a}_{2}\right)} \chi_{31}^{B, d}+\chi_{31}^{B, u},
\end{aligned}
$$

where $H_{0}$ is the bare Hamiltonian, and we use the spinor notation $\psi_{\mathbf{k}}^{\dagger}=\left[a_{1}^{\dagger}(\mathbf{k}), b_{1}^{\dagger}(\mathbf{k}), a_{2}^{\dagger}(\mathbf{k}), b_{2}^{\dagger}(\mathbf{k}), a_{3}^{\dagger}(\mathbf{k}), b_{3}^{\dagger}(\mathbf{k})\right]$. In Fig. 1, we show how the 27 mean-field parameters making up the Fock contribution can be interpreted as NN or NNN hoppings. The six charge-like mean-field parameters come from the Hartree contribution due to charge imbalance between the six sites of the unit cell. Due to charge conservation, only five of them are independent.

\section{Mean-field equations}

Minimizing the free energy functional

$$
\begin{aligned}
F[\xi, \chi, \rho] & =\langle H\rangle_{\mathrm{MF}}-T \mathcal{S}_{\mathrm{MF}}=\mathcal{F}_{\mathrm{MF}}+\left\langle H-H_{\mathrm{MF}}\right\rangle_{\mathrm{MF}} \\
& \simeq \Omega_{\mathrm{MF}}+\left\langle H-H_{\mathrm{MF}}\right\rangle_{\mathrm{MF}}+\mu N_{e},
\end{aligned}
$$

we obtain the following set of mean-field equations:

$$
\begin{aligned}
\xi_{i j} & =-\frac{V_{1}}{N} \sum_{\mathbf{q}} \gamma_{\mathbf{q}}^{i j}\left\langle b_{j, \mathbf{q}}^{\dagger} a_{i, \mathbf{q}}\right\rangle_{\mathrm{MF}}, \\
\chi_{i j, \mathbf{k}}^{A} & =-\frac{V_{2}}{N} \sum_{\mathbf{q}} \alpha_{\mathbf{k}-\mathbf{q}}^{i j}\left\langle a_{j, \mathbf{q}}^{\dagger} a_{i, \mathbf{q}}\right\rangle_{\mathrm{MF}},
\end{aligned}
$$

$$
\begin{aligned}
\chi_{i j, \mathbf{k}}^{B} & =-\frac{V_{2}}{N} \sum_{\mathbf{q}} \beta_{\mathbf{k}-\mathbf{q}}^{i j}\left\langle b_{j, \mathbf{q}}^{\dagger} b_{i, \mathbf{q}}\right\rangle_{\mathrm{MF}}, \\
\rho_{i}^{A} & =V_{1} n^{B}+3 V_{2} n^{A}-3 V_{2} n_{i}^{A}, \\
\rho_{i}^{B} & =V_{1} n^{A}+3 V_{2} n^{B}-3 V_{2} n_{i}^{B},
\end{aligned}
$$

where we have defined $n_{i}=\frac{1}{N} \sum_{\mathbf{q}}\left\langle c_{i, \mathbf{q}}^{\dagger} c_{i, \mathbf{q}}\right\rangle_{\mathrm{MF}}$ and $n=$ $\sum_{i=1}^{3} n_{i}$, with $c=a, b$.

It is easy to show, using Eqs. (4), (5), (A1), and (A2), that the mean-field equation for the effective NNN hoppings can be cast in the $\mathbf{k}$-independent form

$$
\chi_{i j}^{\Gamma, \delta}=-\frac{V_{2}}{N} \sum_{\mathbf{q}} \lambda_{i j, \mathbf{q}}^{\Gamma, \delta}\left\langle c_{j, \mathbf{q}}^{\dagger} c_{i, \mathbf{q}}\right\rangle_{\mathrm{MF}},
$$

with

$$
\begin{gathered}
\lambda_{12, \mathbf{q}}^{A, u}=1 \quad \lambda_{12, \mathbf{q}}^{A, d}=e^{-i \mathbf{q} \cdot\left(\mathbf{a}_{1}+\mathbf{a}_{2}\right)} \lambda_{12, \mathbf{q}}^{A, h}=e^{-i \mathbf{q} \cdot \mathbf{a}_{2}}, \\
\lambda_{23, \mathbf{q}}^{A, u}=e^{i \mathbf{q} \cdot\left(\mathbf{a}_{1}+\mathbf{a}_{2}\right)} \lambda_{23, \mathbf{q}}^{A, d}=1 \quad \lambda_{23, \mathbf{q}}^{A, h}=e^{i \mathbf{q} \cdot \mathbf{a}_{1}}, \\
\lambda_{31, \mathbf{q}}^{A, u}=e^{i \mathbf{q} \cdot \mathbf{a}_{2}} \lambda_{31, \mathbf{q}}^{A, d}=e^{-i \mathbf{q} \cdot \mathbf{a}_{1}} \lambda_{31, \mathbf{q}}^{A, h}=1,
\end{gathered}
$$

and

$$
\begin{gathered}
\lambda_{12, \mathbf{q}}^{B, h}=1 \quad \lambda_{12, \mathbf{q}}^{B, d}=e^{-i \mathbf{q} \cdot \mathbf{a}_{2}} \quad \lambda_{12, \mathbf{q}}^{B, u}=e^{i \mathbf{q} \cdot \mathbf{a}_{1}}, \\
\lambda_{23, \mathbf{q}}^{B, h}=e^{i \mathbf{q} \cdot \mathbf{a}_{2}} \lambda_{23, \mathbf{q}}^{B, d}=1 \quad \lambda_{23, \mathbf{q}}^{B, u}=e^{i \mathbf{q} \cdot\left(\mathbf{a}_{1}+\mathbf{a}_{2}\right)}, \\
\lambda_{31, \mathbf{q}}^{B, h}=e^{-i \mathbf{q} \cdot \mathbf{a}_{1}} \quad \lambda_{31, \mathbf{q}}^{B, d}=e^{-i \mathbf{q} \cdot\left(\mathbf{a}_{1}+\mathbf{a}_{2}\right)} \lambda_{31, \mathbf{q}}^{B, u}=1 .
\end{gathered}
$$

\section{Solving the mean-field equation}

In order to solve the set of mean-field equations given above, we need to compute the averages of the form $\left\langle b_{j}^{\dagger}(\mathbf{q}) a_{i}(\mathbf{q})\right\rangle_{\mathrm{MF}}$. 
To calculate these averages, we introduce the unitary transformation $\mathcal{U}$ that diagonalizes $\mathcal{H}_{\mathrm{MF}}(\mathbf{k})$,

$$
\mathcal{U} \mathcal{H}_{\mathrm{MF}}(\mathbf{k}) \mathcal{U}^{\dagger}=\operatorname{diag}\left[\varepsilon_{1}(\mathbf{k}), \ldots, \varepsilon_{6}(\mathbf{k})\right] .
$$

The new operators $c_{\alpha}(\mathbf{k})$ are such that

$$
\left[\begin{array}{c}
c_{1}(\mathbf{k}) \\
c_{2}(\mathbf{k}) \\
\vdots \\
c_{5}(\mathbf{k}) \\
c_{6}(\mathbf{k})
\end{array}\right]=\mathcal{U}\left[\begin{array}{c}
a_{1}(\mathbf{k}) \\
b_{1}(\mathbf{k}) \\
\vdots \\
a_{3}(\mathbf{k}) \\
b_{3}(\mathbf{k})
\end{array}\right],
$$

and the average $\left\langle b_{j}^{\dagger}(\mathbf{q}) a_{i}(\mathbf{q})\right\rangle_{\mathrm{MF}}$ and $\left\langle c_{j}^{\dagger}(\mathbf{q}) c_{i}(\mathbf{q})\right\rangle_{\mathrm{MF}}$ may be written as

$$
\begin{aligned}
\left\langle b_{j}^{\dagger}(\mathbf{q}) a_{i}(\mathbf{q})\right\rangle_{\mathrm{MF}} & =\left\langle\sum_{\alpha} U_{\alpha, 2 j} c_{\alpha}^{\dagger}(\mathbf{q}) \sum_{\beta} U_{\beta, 2 i-1}^{*} c_{\beta}(\mathbf{q})\right\rangle_{\mathrm{MF}} \\
& =\sum_{\alpha=1}^{6} U_{\alpha, 2 j} U_{\alpha, 2 i-1}^{*}\left\langle c_{\alpha}^{\dagger}(\mathbf{q}) c_{\alpha}(\mathbf{q})\right\rangle_{\mathrm{MF}} \\
& =\sum_{\alpha=1}^{6} U_{\alpha, 2 j} U_{\alpha, 2 i-1}^{*} f\left[\varepsilon_{\alpha}(\mathbf{q})\right]
\end{aligned}
$$

and

$$
\begin{aligned}
\left\langle a_{j}^{\dagger}(\mathbf{q}) a_{i}(\mathbf{q})\right\rangle_{\mathrm{MF}} & =\sum_{\alpha=1}^{6} U_{\alpha, 2 j-1} U_{\alpha, 2 i-1}^{*} f\left[\varepsilon_{\alpha}(\mathbf{q})\right], \\
\left\langle b_{j}^{\dagger}(\mathbf{q}) b_{i}(\mathbf{q})\right\rangle_{\mathrm{MF}} & =\sum_{\alpha=1}^{6} U_{\alpha, 2 j} U_{\alpha, 2 i}^{*} f\left[\varepsilon_{\alpha}(\mathbf{q})\right],
\end{aligned}
$$

and the densities $\left\langle c_{i}^{\dagger}(\mathbf{q}) c_{i}(\mathbf{q})\right\rangle_{\mathrm{MF}}$ as

$$
\begin{aligned}
\left\langle a_{i}^{\dagger}(\mathbf{q}) a_{i}(\mathbf{q})\right\rangle_{\mathrm{MF}} & =\sum_{\alpha=1}^{6}\left|U_{\alpha, 2 i-1}\right|^{2} f\left[\varepsilon_{\alpha}(\mathbf{q})\right], \\
\left\langle b_{i}^{\dagger}(\mathbf{q}) b_{i}(\mathbf{q})\right\rangle_{\mathrm{MF}} & =\sum_{\alpha=1}^{6}\left|U_{\alpha, 2 i}\right|^{2} f\left[\varepsilon_{\alpha}(\mathbf{q})\right],
\end{aligned}
$$

with

$$
f\left[\varepsilon_{\alpha}(\mathbf{q})\right]=\frac{1}{\exp \beta\left[\varepsilon_{\alpha}(\mathbf{q})-\mu\right]+1} .
$$

Then, we can write the set of mean-field equations as

$$
\begin{gathered}
\xi_{i j}=-\frac{V_{1}}{N} \sum_{\mathbf{q}} \gamma_{\mathbf{q}}^{i j} \sum_{\alpha=1}^{6} U_{\alpha, 2 j} U_{\alpha, 2 i-1}^{*} f\left[\varepsilon_{\alpha}(\mathbf{q})\right], \\
\chi_{i j}^{A, \delta}=-\frac{V_{2}}{N} \sum_{\mathbf{q}} \lambda_{i j, \mathbf{q}}^{A, \delta} \sum_{\alpha=1}^{6} U_{\alpha, 2 j-1} U_{\alpha, 2 i-1}^{*} f\left[\varepsilon_{\alpha}(\mathbf{q})\right], \\
\chi_{i j}^{B, \delta}=-\frac{V_{2}}{N} \sum_{\mathbf{q}} \lambda_{i j, \mathbf{q}}^{B, \delta} \sum_{\alpha=1}^{6} U_{\alpha, 2 j} U_{\alpha, 2 i}^{*} f\left[\varepsilon_{\alpha}(\mathbf{q})\right], \\
\rho_{i}^{A}=V_{1} n^{B}+3 V_{2} n^{A}-\frac{3 V_{2}}{N} \sum_{\mathbf{q}} \sum_{\alpha=1}^{6}\left|U_{\alpha, 2 i-1}\right|^{2} f\left[\varepsilon_{\alpha}(\mathbf{q})\right],
\end{gathered}
$$

$$
\rho_{i}^{B}=V_{1} n^{A}+3 V_{2} n^{B}-\frac{3 V_{2}}{N} \sum_{\mathbf{q}} \sum_{\alpha=1}^{6}\left|U_{\alpha, 2 i}\right|^{2} f\left[\varepsilon_{\alpha}(\mathbf{q})\right],
$$

with

$$
\begin{aligned}
& n^{A}=\frac{1}{N} \sum_{\mathbf{q}} \sum_{i=1}^{3} \sum_{\alpha=1}^{6}\left|U_{\alpha, 2 i-1}\right|^{2} f\left[\varepsilon_{\alpha}(\mathbf{q})\right], \\
& n^{B}=\frac{1}{N} \sum_{\mathbf{q}} \sum_{i=1}^{3} \sum_{\alpha=1}^{6}\left|U_{\alpha, 2 i}\right|^{2} f\left[\varepsilon_{\alpha}(\mathbf{q})\right] .
\end{aligned}
$$

This set of equations has to be solved self-consistently with the constrain imposed by the Luttinger theorem, which reads (ignoring logarithmic corrections in fermion number $N_{e}$ ),

$$
\begin{aligned}
3+n \equiv \frac{N_{e}}{N} & =\frac{1}{N} \frac{\partial \Omega}{\partial \mu} \approx \frac{1}{N} \frac{\partial \Omega_{\mathrm{MF}}}{\partial \mu} \\
& =\frac{\langle\mathcal{N}\rangle_{\mathrm{MF}}}{N}=\frac{1}{N} \sum_{\mathbf{q}, \alpha} f\left[\varepsilon_{\alpha}(\mathbf{q})\right],
\end{aligned}
$$

and from which we get $\mu$ self-consistently.

\section{Free energy}

To check the stability of possible phases, we need the free energy defined in Eq. (A3). For a given set of converged order parameters, we have

$$
\mathcal{F}=\Omega_{\mathrm{MF}}+\mu N_{e}+\left\langle\mathcal{H}-\mathcal{H}_{\mathrm{MF}}\right\rangle_{\mathrm{MF}},
$$

where the mean-field grand canonical potential is given by

$$
\Omega_{\mathrm{MF}}=-k_{B} T \sum_{\mathbf{q}, \alpha} \ln \left\{1+e^{-\beta\left[\varepsilon_{\alpha}(\mathbf{q})-\mu\right]}\right\}
$$

and

$$
\begin{aligned}
\left\langle\mathcal{H}-\mathcal{H}_{\mathrm{MF}}\right\rangle_{\mathrm{MF}}= & V_{1} N n_{A} n_{B}-\frac{V_{1}}{N} \sum_{i, j}\left|A_{i j}\right|^{2}-N \sum_{i}\left(\rho_{i}^{A} n_{i}^{A}+\rho_{i}^{B} n_{i}^{B}\right)-\left(\sum_{i, j} \xi_{i j} A_{i j}+\text { c.c. }\right)+\frac{3}{2} V_{2} N n_{A} n_{A}-\frac{3}{2} V_{2} N \sum_{i=1}^{3}\left(n_{i}^{A}\right)^{2} \\
& +\frac{3}{2} V_{2} N n_{B} n_{B}-\frac{3}{2} V_{2} N \sum_{i=1}^{3}\left(n_{i}^{B}\right)^{2}-\frac{V_{2}}{N} \sum_{\Gamma=\{A, B\}} \sum_{\delta=\{u, d, h\}}\left(\left|D_{12}^{\Gamma, \delta}\right|^{2}+\left|D_{23}^{\Gamma, \delta}\right|^{2}+\left|D_{31}^{\Gamma, \delta}\right|^{2}\right) \\
& -\sum_{\Gamma=\{A, B\}} \sum_{\delta=\{u, d, h\}}\left(\chi_{12}^{\Gamma, \delta}\left[D_{12}^{\Gamma, \delta}\right]^{*}+\chi_{23}^{\Gamma, \delta}\left[D_{23}^{\Gamma, \delta}\right]^{*}+\left[\chi_{31}^{\Gamma, \delta}\right]^{*} D_{31}^{\Gamma, \delta}+\text { c.c. }\right),
\end{aligned}
$$


where

$$
\begin{aligned}
A_{i j} & =\sum_{\mathbf{q}, \alpha}\left(\gamma_{\mathbf{q}}^{i j}\right)^{*} U_{\alpha, 2 i-1} U_{\alpha, 2 j}^{*} f\left[\varepsilon_{\alpha}(\mathbf{q})\right], \quad D_{i j}^{A, \delta}=\sum_{\mathbf{q}} \lambda_{i j, \mathbf{q}}^{A, \delta} \sum_{\alpha=1}^{6} U_{\alpha, 2 j-1} U_{\alpha, 2 i-1}^{*} f\left[\varepsilon_{\alpha}(\mathbf{q})\right], \\
D_{i j}^{B, \delta} & =\sum_{\mathbf{q}} \lambda_{i j, \mathbf{q}}^{B, \delta} \sum_{\alpha=1}^{6} U_{\alpha, 2 j} U_{\alpha, 2 i}^{*} f\left[\varepsilon_{\alpha}(\mathbf{q})\right] .
\end{aligned}
$$

${ }^{1}$ F. D. M. Haldane, Phys. Rev. Lett. 93, 206602 (2004).

${ }^{2}$ F. D. M. Haldane, Phys. Rev. Lett. 61, 2015 (1988).

${ }^{3}$ G. E. Volovik, The Universe in a Helium Droplet (Clarendon Press, Oxford, 2003)

${ }^{4}$ X.-G. Wen, Quantum Field Theory of Many-Body Systems (Oxford University Press, Oxford, 2004).

${ }^{5}$ B. A. Bernevig, T. L. Hughes, and S. Zhang, Science 314, 1757 (2006).

${ }^{6}$ M. Z. Hasan and C. L. Kane, Rev. Mod. Phys. 82, 3045 (2010).

${ }^{7}$ X. Qi and S. Zhang, Rev. Mod. Phys. 83, 1057 (2011).

${ }^{8}$ Z. F. Ezawa, Quantum Hall Effects - Field Theoretical Approach and Related Topics (World Scientific, Singapore, 2008).

${ }^{9}$ N. Nagaosa, J. Sinova, S. Onoda, A. H. MacDonald, and N. P. Ong, Rev. Mod. Phys. 82, 1539 (2010).

${ }^{10}$ K. Sun and E. Fradkin, Phys. Rev. B 78, 245122 (2008).

${ }^{11}$ A. Cortijo, A. G. Grushin, and M. A. H. Vozmediano, Phys. Rev. B 82, 195438 (2010).

${ }^{12}$ A. Cortijo, F. Guinea, and M. A. H. Vozmediano, J. Phys. A 45, 383001 (2012).

${ }^{13}$ G. V. Semenoff, Phys. Rev. Lett. 53, 2449 (1984).

${ }^{14}$ C. Kane and E. Mele, Phys. Rev. Lett. 95, 226801 (2005).

${ }^{15}$ C. Kane and E. Mele, Phys. Rev. Lett. 95, 146802 (2005).

${ }^{16}$ D. A. Pesin and L. Balents, Nat. Phys. 6, 376 (2010).

${ }^{17}$ C. N. Varney, K. Sun, M. Rigol, and V. Galitski, Phys. Rev. B 84, 241105(R) (2011).

${ }^{18}$ S. Rachel and K. L. Hur, Phys. Rev. B 82, 075106 (2010).

${ }^{19}$ D. Soriano and J. Fernández-Rossier, Phys. Rev. B 82, 161302 (2010).

${ }^{20}$ M. Hohenadler, T. C. Lang, and F. F. Assaad, Phys. Rev. Lett. 106, 100403 (2011).

${ }^{21}$ D. Zheng, G.-M. Zhang, and C. Wu, Phys. Rev. B 84, 205121 (2011).

${ }^{22}$ L. Wang, H. Shi, S. Zhang, X. Wang, X. Dai, and X. C. Xie, arXiv:1012.5163v1 (unpublished).

${ }^{23}$ D.-H. Lee, Phys. Rev. Lett. 107, 166806 (2011).

${ }^{24}$ M. A. N. Araújo, E. V. Castro, and P. D. Sacramento, Phys. Rev. B 87, 085109 (2013).

${ }^{25}$ S. Raghu, X.-L. Qi, C. Honerkamp, and S.-C. Zhang, Phys. Rev. Lett. 100, 156401 (2008).

${ }^{26}$ K. Sun, H. Yao, E. Fradkin, and S. A. Kivelson, Phys. Rev. Lett. 103, 046811 (2009).
${ }^{27}$ Q. Liu, H. Yao, and T. Ma, Phys. Rev. B 82, 045102 (2010).

${ }^{28}$ J. Wen, A. Ruegg, C.-C. J. Wang, and G. A. Fiete, Phys. Rev. B 82, 075125 (2010).

${ }^{29}$ C. Weeks and M. Franz, Phys. Rev. B 81, 085105 (2010).

${ }^{30} \mathrm{~T}$. Li, arXiv:1101.1352 (unpublished).

${ }^{31}$ R. Nandkishore, L. Levitov, and A. Chubukov, Nat. Phys. 8, 158 (2012).

${ }^{32}$ E. V. Castro, A. G. Grushin, B. Valenzuela, M. A. H. Vozmediano, A. Cortijo, and F. de Juan, Phys. Rev. Lett. 107, 106402 (2011).

${ }^{33}$ S. Zhu, B. Wang, and L. Duan, Phys. Rev. Lett. 98, 260402 (2007).

${ }^{34}$ J. Mañes, F. Guinea, and M. A. H. Vozmediano, Phys. Rev. B 75, 155424 (2007).

${ }^{35}$ C.-Y. Hou, C. Chamon, and C. Mudry, Phys. Rev. Lett. 98, 186809 (2007).

${ }^{36}$ J. L. McChesney et al., Phys. Rev. Lett. 104, 136803 (2010).

${ }^{37}$ B. Valenzuela and M. A. H. Vozmediano, New J. Phys. 10, 113009 (2008).

${ }^{38}$ D. Makogon, R. van Gelderen, R. Roldán, and C. Morais Smith, Phys. Rev. B 84, 125404 (2011).

${ }^{39}$ G. Murthy, E. Shimshoni, R. Shankar, and H. A. Fertig, Phys. Rev. B 85, 073103 (2012).

${ }^{40}$ A. G. Grushin, Ph.D. thesis, Instituto de Ciencia de Materiales de Madrid, 2013 (unpublished).

${ }^{41}$ S. Gopalakrishnan, P. Ghaemi, and S. Ryu, Phys. Rev. B 86, 081403(R) (2012).

${ }^{42}$ B. Valenzuela, S. Fratini, and D. Baeriswyl, Phys. Rev. B 68, 045112 (2003).

${ }^{43}$ S. Fratini and J. Merino, Phys. Rev. B 80, 165110 (2009).

${ }^{44}$ R. Nandkishore, G. Chern, and A. V. Chubukov, Phys. Rev. Lett. 108, 227204 (2012).

${ }^{45}$ C. Wu, Phys. Rev. Lett. 101, 186807 (2012).

${ }^{46}$ L. B. Shao, S. Zhu, L. Sheng, D. Y. Xing, and Z. D. Wang, Phys. Rev. Lett. 101, 246810 (2008).

${ }^{47}$ K. L. Lee, B. Gremaud, R. Han, B. Englert, and C. Miniatura, Phys. Rev. A 80, 043411 (2009).

${ }^{48}$ A. Dauphin, M. Muller, and M. A. Martin-Delgado, Phys. Rev. A 86, 053618 (2012).

${ }^{49}$ L. Tarruell, D. Greif, T. Uehlinger, G. Jotzu, and T. Esslinger, Nature (London) 483, 302 (2012). 